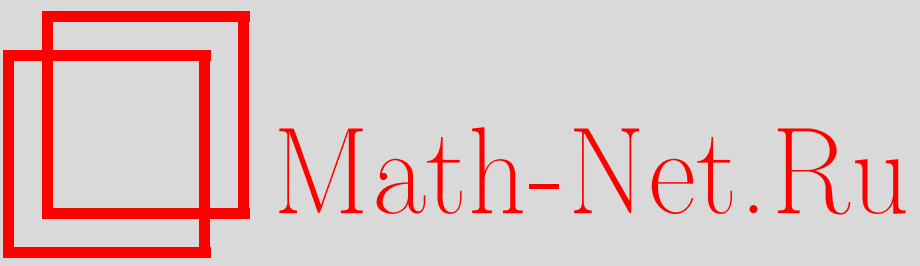

А. С. Чихачев, Связанные состояния частицы в поле разбегающихся точечных потенциалов, ТМФ, 2005, том 145, номер 3, 385-392

DOI: https://doi.org/10.4213/tmf1907

Использование Общероссийского математического портала Math-Net.Ru подразумевает, что вы прочитали и согласны с пользовательским соглашением

http://www.mathnet.ru/rus/agreement

Параметры загрузки:

IP: 54.198 .64 .247

26 апреля 2023 г., 14:51:06 
ТЕОРЕТИЧЕСКАЯ

И МАТЕМАТИЧЕСКАЯ

ФИЗИКА

Том 145, № 3

декабрь, 2005

(C) 2005 г.

А.С. Чихачев*

\section{СВЯЗАННЫЕ СОСТОЯНИЯ ЧАСТИЦЫ В ПОЛЕ РАЗБЕГАЮШИХСЯ ТОЧЕЧНЫХ ПОТЕНЦИАЛОВ}

Изучаются связанные состояния в нестационарной системе разбегающихся $\delta$-центров. Рассмотрены состояния, характеризующиеся разной глубиной уровня точечных центров, в одномерной и трехмерной задачах.

Ключевые слова: уравнение Шредингера, точечный потенциал, связанное состояние.

\section{1. ВВЕДЕНИЕ}

Представление о потенциале нулевого радиуса действия оказывается плодотворным для решения нестационарных задач квантовой механики. При помощи нестационарных моделей с точечными взаимодействиями, допускающих точное решение, изучались нуклонное туннелирование [1], нейтрализация и перезарядка атомных частиц [2], [3]. Большое число работ посвяшено изучению рассеяния частиц на движущихся точечных центрах (см., например, работы [4]). Ряд работ посвящен решению различных вопросов, связанных с поиском точного решения нестационарного уравнения Шредингера [5]-[7]. При этом связанные состояния в системе разбегаюшихся центров представляются недостаточно изученными. Под “связанными” имеются в виду состояния, описьваемые функциями, достаточно быстро (экспоненциально) убываюшими по пространственным переменным. По-видимому, впервые связанное состояние в системе разбегаюшихся центров в одномерной задаче рассматривалось в работе [5]. В настоящей работе изучены связанные состояния для одномерной и трехмерной систем, причем рассматриваются системы, характеризуемые разной глубиной уровня разбегающихся центров. Решения определяются при помощи опережающей функции Грина.

\section{2. СИММЕТРИЧНАЯ ЗАДАЧА В ОДНОМЕРНОЙ МОДЕЛИ}

Рассмотрим одномерное уравнение Шредингера следующего вида:

$$
i \frac{\partial \psi}{\partial t}+\frac{1}{2} \frac{\partial^{2} \psi}{\partial x^{2}}=-\alpha[\delta(x-v t)+\delta(x+v t)] \psi(x, t) .
$$

* Всероссийский электротехнический институт им. В.И. Ленина, Москва, Россия. E-mail: churchev@mail.ru 
Здесь $\psi(x, t)$ - пси-функция, используется система единиц, в которой $m=\hbar=e=1$. При $v=0$ уравнение (1) имеет решение, описываюшее единственное связанное состояние:

$$
\psi(x, t)=\text { const } \cdot e^{-2 \alpha|x|} e^{2 i \alpha^{2} t} .
$$

Опережающая функция Грина свободного уравнения Шредингера имеет вид

$$
G_{0}^{(-)}=\frac{-\sigma\left(t^{\prime}-t\right)}{\sqrt{-2 \pi i\left(t-t^{\prime}\right)}} \exp \left\{-\frac{i\left(x-x^{\prime}\right)^{2}}{2\left(t^{\prime}-t\right)}\right\}
$$

где $\sigma(x)=1$ при $x \geqslant 0, \sigma(x)=0$ при $x<0$.

Уравнение (1) с учетом (2) может быть представлено в интегральной форме

$$
\psi(x, t)=\frac{\alpha}{\sqrt{2 \pi i}} \int_{t}^{\infty} \frac{d t^{\prime}}{\sqrt{t^{\prime}-t}}\left\{\exp \left[-\frac{i(x-v t)^{2}}{2\left(t^{\prime}-t\right)}\right]+\exp \left[-\frac{(x+v t)^{2}}{2\left(t^{\prime}-t\right)}\right]\right\} \psi\left(v t^{\prime}, t\right) .
$$

Рассмотрим симметричный случай $\psi(x, t)=\psi(-x, t)$. Если обозначить $f(t)=$ $e^{-i v^{2} t / 2} \psi(v t, t)$, то из $(3)$ следует интегральное уравнение для $f:$

$$
f=\frac{\alpha}{\sqrt{2 \pi i}} \int_{t}^{\infty} \frac{d t^{\prime} f\left(t^{\prime}\right)}{\sqrt{t^{\prime}-t}}\left(1+\exp \left[-\frac{2 i v^{2} t t^{\prime}}{t^{\prime}-t}\right]\right)
$$

Это уравнение может быть приведено к уравнению с разностным ядром посредством замены $t=\tau^{-1}, t^{\prime}=\left(\tau^{\prime}\right)^{-1}, g(\tau) \equiv f\left(\tau^{-1}\right) \tau^{-3 / 2}$. Для образа Лапласа

$$
h(p)=\int_{0}^{\infty} g(\tau) e^{-p \tau} d \tau
$$

можно получить дифференциальное уравнение первого порядка, для решения которого удобно положить $p=q^{2} /(2 i)$. Решение имеет вид

$$
h(q)=C_{0} \exp \left(i \alpha q-\frac{i \alpha}{2 v} e^{-2 v q}+\frac{i \alpha}{2 v}\right)
$$

где множитель $e^{i \alpha /(2 v)}$ обеспечивает плавный переход к случаю $v \rightarrow 0$ (при этом $C_{0}$ не зависит от $v$ ). Обратное преобразование Лапласа позволяет получить следующее решение для $f(t)$ :

$$
f(t)=-\frac{C_{0}}{2 \pi t^{3 / 2}} \int_{i \infty}^{\infty} q \exp \left\{-\frac{i q^{2}}{2 t}+\frac{i \alpha}{2 v}-\frac{i \alpha}{2 v} e^{-2 v q}+i \alpha q\right\} d q .
$$

Контур интегрирования в комплексной плоскости $q$ представляет собой два луча $(+i \infty, 0)$ и $(0,+\infty)$. Использование разложения

$$
\exp \left(-\frac{i \alpha}{2 v} e^{-2 v q}\right)=\sum_{s} \frac{1}{s !}\left(-\frac{i \alpha}{2 v}\right)^{s} e^{-2 s v q}
$$

приводит к равенству

$$
f(t)=\frac{C_{0}}{\sqrt{2 \pi i}} \sum_{s} \frac{1}{s !}\left(-\frac{i \alpha}{2 v}\right)^{s}(\alpha+2 i v s) \exp \left(\frac{i t}{2}(\alpha+2 i v s)^{2}\right) e^{i \alpha /(2 v)}
$$


Чтобы получить решение уравнения (1) в явном виде, следует вычислить интеграл $\psi(x, t)=\frac{\alpha}{\sqrt{2 \pi i}} \int_{t}^{\infty} \frac{d t^{\prime}}{\sqrt{t^{\prime}-t}} f\left(t^{\prime}\right) e^{-i v^{2} t^{\prime} / 2}\left(\exp \left(-\frac{i\left(x-v t^{\prime}\right)^{2}}{2\left(t^{\prime}-t\right)}\right)+\exp \left(-\frac{i(x+v t)^{2}}{2\left(t^{\prime}-t\right)}\right)\right)$.

В результате получим

$$
\begin{aligned}
\psi(x, t)= & \sum_{s} B_{s} \exp \left(\frac{i t(\alpha+2 i v s)^{2}}{2}-\frac{i v^{2} t}{2}\right) \times \\
& \times\{\exp (i v x-(\alpha+2 i v s)|x-v t|)+\exp (-i v x-(\alpha+2 i v s)|x+v t|)\},
\end{aligned}
$$

где

$$
B_{s}=\frac{\alpha C_{0}}{\sqrt{2 \pi i}}\left(-\frac{i \alpha}{2 v}\right)^{s} e^{i \alpha / 2 v} \frac{1}{s !} .
$$

Выражение (8) совпадает с решением, полученным другим методом в работе [5].

Решение (8) является симметричным по $x$ решением уравнения (1). Если в (8) сумму экспонент в фигурных скобках заменить на их разность, то полученное выражение также будет удовлетворять уравнению (1). Это решение, антисимметричное по $x$, найдено в работе [2]. Отметим, что если симметричное решение при $v \rightarrow 0$ переходит в связанное состояние одного $\delta$-центра с удвоенной константой связи, то антисимметричное решение при $v \rightarrow 0$ переходит в $\psi \equiv 0$.

\section{3. ОДНОМЕРНАЯ МОДЕЛЬ ДЛЯ ЦЕНТРОВ С РАЗНОЙ ГЛУБИНОЙ}

Рассмотрим несимметричный случай - разбегание $\delta$-центров, характеризующихся различной глубиной связанного уровня. Уравнение Шредингера

$$
i \frac{\partial \psi}{\partial t}+\frac{1}{2} \frac{\partial^{2} \psi}{\partial x^{2}}=-[\alpha \delta(x-v t)+\beta \delta(x+v t)] \psi
$$

в интегральной форме имеет вид

$$
\psi=\frac{1}{\sqrt{2 \pi i}} \int_{t}^{\infty} \frac{d t^{\prime}}{\sqrt{t^{\prime}-t}}\left(\alpha f_{1}\left(t^{\prime}\right) \exp \left(-\frac{i\left(x-v t^{\prime}\right)^{2}}{2\left(t^{\prime}-t\right)}\right)+\beta f_{2}\left(t^{\prime}\right) \exp \left(-\frac{i\left(x+v t^{\prime}\right)^{2}}{2\left(t^{\prime}-t\right)}\right)\right),
$$

где $f_{1}=e^{-i v^{2} t / 2} \psi(v t, t), f_{2}=e^{-i v^{2} t / 2} \psi(-v t, t)$. Вместо одного уравнения (4) получим систему

$$
\begin{aligned}
& f_{1}(t)=\frac{\alpha}{\sqrt{2 \pi i}} \int_{t}^{\infty} \frac{d t^{\prime}}{\sqrt{t^{\prime}-t}} f_{1}\left(t^{\prime}\right)+\frac{\beta}{\sqrt{t^{\prime}-t}} \int_{t}^{\infty} \frac{d t^{\prime}}{\sqrt{t^{\prime}-t}} f_{2}\left(t^{\prime}\right) \exp \left(-\frac{2 i v^{2} t t^{\prime}}{t^{\prime}-t}\right) \\
& f_{2}(t)=\frac{\alpha}{\sqrt{2 \pi i}} \int_{t}^{\infty} \frac{d t^{\prime}}{\sqrt{t^{\prime}-t}} f_{1}\left(t^{\prime}\right) \exp \left(-\frac{2 i v^{2} t t^{\prime}}{t^{\prime}-t}\right)+\frac{\beta}{\sqrt{2 \pi i}} \int_{t}^{\infty} \frac{d t^{\prime}}{\sqrt{t^{\prime}-t}} f_{2}\left(t^{\prime}\right) .
\end{aligned}
$$

Положим $h_{1,2}=\int_{0}^{\infty} g_{1,2}(\tau) e^{-p \tau} d \tau$, где $g_{1,2}=f_{1,2}\left(\tau^{-1}\right) \tau^{-3 / 2}$. Для $h_{1,2}(q)$ (при $p=$ $\left.q^{2} /(2 i)\right)$ получим систему уравнений

$$
\begin{aligned}
-\frac{i}{q} \frac{d h_{1}}{d q} & =\frac{\alpha}{q} h_{1}+\frac{\beta}{q} h_{2} e^{-2 v q} \\
-\frac{i}{q} \frac{d h_{2}}{d q} & =\frac{\alpha}{q} h_{1} e^{-2 v q}+\frac{\beta}{q} h_{2} .
\end{aligned}
$$


Система (10) имеет решение следующего вида:

$$
\begin{aligned}
& h_{1}=e^{(-v-i(\alpha+\beta) / 2)}\left\{C_{1} J_{\frac{1}{2}+i \frac{\alpha-\beta}{4 v}}(z)+C_{2} J_{\frac{1}{2}-i \frac{\alpha-\beta}{4 v}}(z)\right\}, \\
& h_{2}=e^{(-v-i(\alpha+\beta) / 2)}\left\{C_{3} J_{\frac{1}{2}-i \frac{\alpha-\beta}{4 v}}(z)+C_{4} J_{\frac{1}{2}-i \frac{\alpha-\beta}{4 v}}(z)\right\},
\end{aligned}
$$

где $z=\sqrt{\alpha \beta} e^{-2 v q} /(2 v), J_{\nu}(z)-$ функция Бесселя, $C_{i}$ - произвольные константы. В случае $\alpha=\beta$ и симметричного состояния $h_{1} \equiv h_{2}$ при $C_{1}=i C_{2}$ можно получить, что

$$
h_{1}=h_{2}=\sqrt{\frac{2}{\pi} \frac{2 v}{\alpha}} \exp \left\{-i \alpha q-e^{-2 v q} \frac{i \alpha}{2 v}\right\} C_{*} .
$$

При $C_{*} \sim e^{i \alpha /(2 v)}$ решение соответствует случаю, рассмотренному в предыдушем разделе. В обшем случае разложение функций Бесселя в ряды позволяет получить решение, описьваюшее связанное состояние, в следуюшем виде:

$$
\psi(x, t)=e^{-i v^{2} t / 2} \sum_{s}\left\{C_{s}^{(1)} \varphi_{s}\left(z_{-}, t\right) e^{i v x}+C_{s}^{(2)} \chi_{s}\left(z_{+}, t\right) e^{-i v x}\right\},
$$

где $z_{ \pm}=|x \pm v t|, \varphi_{s}=\exp \left(-a_{s} z+i a_{s}^{2} t / 2\right), \chi_{s}=\exp \left(-b_{s} z+i b_{s}^{2} t / 2\right)$. Величины $a_{s}$ и $b_{s}$ определяются соотношениями

$a_{2 s+1}=\beta+2 i v(2 s+1), \quad b_{2 s+1}=\alpha+2 i v(2 s+1), \quad a_{2 s}=\alpha+4 i v s, \quad b_{2 s}=\beta+4 i v s$.

Коэффицииенты $C^{(1)}$ имеют вид

$$
C_{2 l}^{(1)}=\left(-\frac{\alpha \beta}{16 v^{2}}\right) C_{0} M_{l}, \quad C_{2 l+1}^{(1)}=\frac{\alpha}{4 i v}\left(-\frac{\alpha \beta}{16 v^{2}}\right) C_{0} N_{l},
$$

где

$$
M_{l}=\frac{1}{l !} \frac{\Gamma\left(\frac{1}{2}+\frac{\alpha-\beta}{4 i v}\right)}{\Gamma\left(\frac{1}{2}+l+\frac{\alpha-\beta}{4 i v}\right)}, \quad N_{l}=\frac{1}{l !} \frac{\Gamma\left(\frac{1}{2}-\frac{\alpha-\beta}{4 i v}\right)}{\Gamma\left(\frac{3}{2}+l-\frac{\alpha-\beta}{4 i v}\right)} .
$$

Коэффициенты $C^{(2)}$ получаются из $C^{(1)}$ заменой $\alpha$ на $\beta$ и $\beta$ на $\alpha$. Справедливость выражения (11) может быть проверена непосредственной подстановкой в уравнение. Это решение также было найдено в работе [6].

\section{4. ТРЕХМЕРНАЯ СИММЕТРИЧНАЯ ЗАДАЧА}

В трехмерном случае одного движушегося с постоянной скоростью $\delta$-центра связанное состояние описывается $\psi$-функцией следуюшего вида:

$$
\psi=\frac{1}{|\mathbf{r}-\mathbf{v} t|} \exp \left\{-\kappa|\mathbf{r}-\mathbf{v} t|+i \mathbf{v}(\mathbf{r}-\mathbf{v} t)+i \frac{v^{2} t}{2}+i \frac{\kappa^{2} t}{2}\right\}
$$

Эта функция удовлетворяет уравнению Шредингера

$$
i \frac{\partial \psi}{\partial t}+\frac{1}{2} \Delta \psi=\frac{2 \pi}{\kappa} \delta(\mathbf{r}-\mathbf{v} t)[(1-i \mathbf{v}(\mathbf{r}-\mathbf{v} t)) \psi+(\mathbf{r}-\mathbf{v} t) \nabla \psi] .
$$


Константа $\kappa$ характеризует глубину связанного уровня. Решение уравнения (13) может быть найдено с помошью опережаюшей функции Грина

$$
G_{0}^{(-)}=\frac{\sigma\left(t^{\prime}-t\right)}{\left[2 \pi i\left(t^{\prime}-t\right)\right]^{3 / 2}} \exp \left\{-\frac{i\left(\mathbf{r}-\mathbf{r}^{\prime}\right)^{2}}{2\left(t^{\prime}-t\right)}\right\}
$$

Правая часть уравнения (13) не равна нулю только в точке $\mathbf{r}=\mathbf{v} t$, а в окрестности этой точки $\psi \sim c(t)(1 /|\mathbf{r}-\mathbf{v} t|-\kappa+i \mathbf{v}(\mathbf{r}-\mathbf{v} t) /|\mathbf{r}-\mathbf{v} t|)$.

Из (13) следует, что

$$
\psi=-\frac{2 \pi}{(2 \pi i)^{3 / 2}} \int_{t}^{\infty} \frac{d t^{\prime} c\left(t^{\prime}\right)}{\left(t^{\prime}-t\right)^{3 / 2}}\left\{\exp \left[-\frac{i\left(\mathbf{r}-\mathbf{v} t^{\prime}\right)^{2}}{2\left(t^{\prime}-t\right)}\right]\right\} .
$$

Если $c(t)=\exp \left\{i \kappa^{2} t / 2+i v^{2} t / 2\right\}$, то из (14) следует соотношение (12), т.е. выполнены граничные условия для $\psi$-функции в точке, где находится $\delta$-центр.

В случае разбегающихся центров уравнение Шредингера имеет вид

$$
\begin{aligned}
i \frac{\partial \psi}{\partial t}+\frac{1}{2} \Delta \psi= & \frac{2 \pi}{\kappa}\{\delta(\mathbf{r}-\mathbf{v} t)[(1-i \mathbf{v}(\mathbf{r}-\mathbf{v} t)) \psi+(\mathbf{r}-\mathbf{v} t) \nabla \psi]+ \\
& +\delta(\mathbf{r}+\mathbf{v} t)[(1+i \mathbf{v}(\mathbf{r}+\mathbf{v} t)) \psi+(\mathbf{r}+\mathbf{v} t) \nabla \psi]\}
\end{aligned}
$$

С учетом того, что $\mathbf{r}= \pm \mathbf{v} t$,

$$
\psi \cong c(t)\left(\frac{1}{|\mathbf{r} \pm \mathbf{v} t|}-\kappa \mp \frac{i \mathbf{v}(\mathbf{r} \pm \mathbf{v} t)}{|\mathbf{r} \pm \mathbf{v} t|}\right)
$$

из (15) следует, что

$$
\psi=-\frac{2 \pi}{(2 \pi i)^{3 / 2}} \int_{t}^{\infty} \frac{d t^{\prime} c\left(t^{\prime}\right)}{\left(t^{\prime}-t\right)^{3 / 2}}\left\{\exp \left[-\frac{i\left(\mathbf{r}-\mathbf{v} t^{\prime}\right)^{2}}{2\left(t^{\prime}-t\right)}\right]+\exp \left[-\frac{i\left(\mathbf{r}+\mathbf{v} t^{\prime}\right)^{2}}{2\left(t^{\prime}-t\right)}\right]\right\} .
$$

Для того чтобы получить уравнение для $c(t)$, вычислим $\{[1-i \mathbf{v}(\mathbf{r}-\mathbf{r} t)+(\mathbf{r}-$ $\mathbf{r} t) \nabla] \psi\}\left.\right|_{\mathbf{r} \rightarrow \mathbf{v} t}$. Имеем

$$
\begin{aligned}
-\kappa c(t)= & -\frac{2 \pi}{(2 \pi i)^{3 / 2}} \int_{t}^{\infty} \frac{d t^{\prime} c\left(t^{\prime}\right)}{\left(t^{\prime}-t\right)^{3 / 2}}\left\{\left[\left(1-i \mathbf{v}(\mathbf{r}-\mathbf{v} t)-\frac{i(\mathbf{r}-\mathbf{v} t)\left(\mathbf{r}-\mathbf{v} t^{\prime}\right)}{t^{\prime}-t}\right) \times\right.\right. \\
& \left.\left.\times \exp \left(-\frac{i\left(\mathbf{r}-\mathbf{v} t^{\prime}\right)^{2}}{2\left(t^{\prime}-t\right)}\right)\right]\left.\right|_{\mathbf{r} \rightarrow \mathbf{v} t}+\exp \left(-\frac{i v^{2}\left(t^{\prime}+t\right)^{2}}{2\left(t^{\prime}-t\right)}\right)\right\} .
\end{aligned}
$$

Положим $c(t)=g(t) e^{i v^{2} t / 2}$. Первое слагаемое, заключенное в квадратные скобки, прежде чем преходить к пределу $\mathbf{r}=\mathbf{v} t$, проинтегрируем по частям. Подстановку

$$
\left.\frac{2}{\left(t^{\prime}-t\right)^{3 / 2}} \exp \left\{\frac{i v^{2} t}{2}-\frac{i(\mathbf{r}-\mathbf{v} t)^{2}}{2\left(t^{\prime}-t\right)}\right\} g\left(t^{\prime}\right)\right|_{t^{\prime}=t} ^{t^{\prime}=\infty}
$$

будем считать равной нулю. Если записать показатель экспоненты в размерных переменных, то в знаменателе появится параметр $\hbar$. Следует сделать замену $\hbar \rightarrow \hbar(1+i \varepsilon)$, 
где $\varepsilon$ - малая положительная величина. При вычислении подстановки при $t^{\prime}>t$ следует сначала устремить $t^{\prime}$ к $t$, а затем положить $\varepsilon=0$. Отметим, что эта процедура эквивалентна процедуре "вычитания бесконечностей”, описанной в книге [8].

В результате получим уравнение для $g(t)$ :

$$
g(t)=-\frac{2 i}{\kappa \sqrt{2 \pi i}} \int_{t}^{\infty} \frac{\dot{g}\left(t^{\prime}\right) d t^{\prime}}{\sqrt{t^{\prime}-t}}-\frac{i}{\kappa \sqrt{2 \pi i}} \int_{t}^{\infty} \frac{g\left(t^{\prime}\right) d t^{\prime}}{\left(t^{\prime}-t\right)^{3 / 2}} \exp \left(\frac{-2 i v^{2} t}{t^{\prime}-t}\right) .
$$

Делая замену $t=\tau^{-1}, t^{\prime}=\left(\tau^{\prime}\right)^{-1}, g\left(\tau^{-1}\right)=h(\tau) \sqrt{\tau}$, можно переписать это уравнение в виде

$$
h(\tau)=\frac{i}{\kappa \sqrt{2 \pi i}} \int_{0}^{\tau} \frac{d \tau^{\prime}\left(2 \dot{h}\left(\tau^{\prime}\right) \tau^{\prime}+h\left(\tau^{\prime}\right)\right)}{\sqrt{\tau-\tau^{\prime}}}-\frac{i \tau}{\kappa \sqrt{2 \pi i}} \int_{0}^{\tau} \frac{d \tau^{\prime} h\left(\tau^{\prime}\right) \exp \left(\frac{-2 i v^{2}}{\tau-\tau^{\prime}}\right)}{\left(\tau-\tau^{\prime}\right)^{3 / 2}} .
$$

Данное интегральное уравнение характеризуется ядром, зависяшим от разности, и может быть решено при помощи преобразования Лапласа. Если положить

$$
H(p)=\int_{0}^{\infty} e^{-p \tau} h(\tau) d \tau
$$

то можно получить для $H(p)$ дифференциальное уравнение первого порядка

$$
\kappa H(p)=-\frac{i}{\sqrt{2 i p}}\left(H(p)+2 p \frac{d H}{d p}\right)+\frac{1}{2 v} \frac{d}{d p}\left(H(p) e^{-v q \sqrt{2 i p}}\right) .
$$

Положим $p=q^{2} /(2 i)$. Тогда уравнение для $H$ можно записать следующим образом:

$$
i \kappa H(q) q=a^{\prime}(q) H+a(q) H^{\prime}
$$

где $a(q)=q-e^{-2 v q} /(2 v), a^{\prime}=d a / d q=1+e^{-2 v q}$.

Решение уравнения (19) имеет вид

$$
H=\frac{\text { const }}{a(q)} \exp \left(i \kappa \int_{0}^{q} \frac{q^{\prime} d q^{\prime}}{a\left(q^{\prime}\right)}\right) .
$$

При этом $g(t)$ определяется равенством

$$
g(t)=\frac{\text { const }}{\sqrt{t}} \int_{L} e^{-i q^{2} /(2 t)} q H(q) d q
$$

Контур $L$ представляет собой лучи $(i \infty, 0)$ и $(0,+\infty)$. Поскольку $c(t)=g(t) e^{i v^{2} t / 2}$, из (21) следует, что

$$
\begin{aligned}
\psi(\mathbf{r}, t)= & \operatorname{const} \int_{t}^{\infty} \frac{d t^{\prime} e^{i v^{2} t^{\prime} / 2}}{\left(t^{\prime}-t\right)^{3 / 2}} \frac{1}{\sqrt{t^{\prime}}} \int_{L} d q q e^{i q^{2} /\left(2 t^{\prime}\right)} \frac{1}{a(q)} \exp \left(i \kappa \int_{0}^{q} \frac{q^{\prime} d q^{\prime}}{a\left(q^{\prime}\right)}\right) \times \\
& \times\left\{\exp \left(-\frac{i\left(\mathbf{r}-\mathbf{v} t^{\prime}\right)^{2}}{2\left(t^{\prime}-t\right)}\right)+\exp \left(-\frac{i\left(\mathbf{r}+\mathbf{v} t^{\prime}\right)^{2}}{2\left(t^{\prime}-t\right)}\right)\right\} \equiv \operatorname{const}\left\{\psi_{-}+\psi_{+}\right\}
\end{aligned}
$$

где

$$
\psi_{ \pm}=\int_{t}^{\infty} \frac{d t^{\prime} e^{i v^{2} t^{\prime} / 2}}{\left(t^{\prime}-t\right)^{3 / 2} \sqrt{t^{\prime}}} \int_{L} d q q e^{-i q^{2} /\left(2 t^{\prime}\right)} \frac{1}{a(q)} \exp \left(i \kappa \int_{0}^{q} \frac{q^{\prime} d q^{\prime}}{a\left(q^{\prime}\right)}\right) \exp \left(-\frac{i\left(\mathbf{r} \pm \mathbf{v} t^{\prime}\right)^{2}}{2\left(t^{\prime}-t\right)}\right) .
$$


Найдем эти функции. Если воспользоваться равенством

$$
\frac{1}{\sqrt{t^{\prime}}} e^{-i q^{2} /\left(2 t^{\prime}\right)}=\frac{1}{\sqrt{2 \pi i}} \int d p e^{i p^{2} t^{\prime} / 2-i p q},
$$

то можно вычислить интеграл по времени:

$$
\begin{aligned}
& \frac{1}{\sqrt{2 \pi i}} \int_{t}^{\infty} \frac{d t^{\prime}}{\left(t^{\prime}-t\right)^{3 / 2}} \exp \left\{\frac{i v^{2} t^{\prime}}{2}+\frac{i p^{2} t^{\prime}}{2}-\frac{i\left(\mathbf{r} \pm \mathbf{v} t^{\prime}\right)^{2}}{2\left(t^{\prime}-t\right)}\right\}= \\
& \quad=\frac{1}{i|\mathbf{r} \pm \mathbf{v} t|} \exp \left\{\mp i \mathbf{v}(\mathbf{r} \pm \mathbf{v} t)+\frac{i p^{2} t}{2}+\frac{i v^{2} t}{2}-p|\mathbf{r} \pm \mathbf{v} t|\right\}
\end{aligned}
$$

Далее, может быть вычислен интеграл по $p=q^{2} /(2 i)$ :

$$
\int d p e^{i p^{2} t / 2-i p q-p r_{ \pm}}=\sqrt{\frac{2 \pi i}{t}} e^{-i\left(q-i r_{ \pm}\right)^{2} /(2 t)},
$$

где $r_{ \pm}=|\mathbf{r} \pm \mathbf{v} t|$. В результате, опуская несушественный постоянный множитель, получим

$$
\psi_{ \pm}=\frac{\exp \left(i v^{2} t / 2 \pm i \mathbf{v} \mathbf{r}_{ \pm}\right)}{r_{ \pm} \sqrt{t}} \int_{L} \frac{q d q}{a(q)} \exp \left\{-\frac{i\left(q-i r_{ \pm}\right)^{2}}{2 t}+i \kappa \int_{0}^{q} \frac{q^{\prime} d q^{\prime}}{a\left(q^{\prime}\right)}\right\}
$$

Непосредственная подстановка найденных функций в уравнение (15) показывает, что $\psi_{-}+\psi_{+}$удовлетворяет этому уравнению. Отметим, что контур интегрирования $L$ в комплексной плоскости $q=q_{1}+i q_{2}$ должен огибать сверху полюс в точке $q: 2 v q=$ $e^{-2 v q}$

Решение (22) аналогично решению (8) для связанного состояния в одномерной задаче. В обоих случаях решение представляет собой сумму двух выражений, зависяших соответственно от $r_{-}$и $r_{+}$.

Для трехмерной задачи, однако, связанное состояние не может быть представлено в виде ряда "метастабильных" затухающих уровней. Кроме того, в отличие от одномерной задачи, в пределе $v \rightarrow 0$ состояние не переходит непрерывным образом в связанное состояние одного центра. Это объясняется, по-видимому, наличием операции “вычитания бесконечностей". При этом в стационарной задаче при стремлении к нулю расстояния между центрами состояние не переходит в связанное состояние одного центра.

\section{5. РАЗБЕГАЮШИЕСЯ ЦЕНТРЫ С РАЗНОЙ ГЛУБИНОЙ УРОВНЯ В ТРЕХМЕРНОЙ ЗАДАЧЕ}

Так же как и в случае одномерной задачи, для трехмерного случая разбегаюшихся центров с разной глубиной уровня задача определения связанного состояния может быть сведена к решению системы обыкновенных дифференциальных уравнений. Вместо соотношения (16) для $\psi$-функции имеет место соотношение

$$
\psi=-\frac{2 \pi}{(2 \pi i)^{3 / 2}} \int_{t}^{\infty} \frac{d t^{\prime}}{\left(t^{\prime}-t\right)^{3 / 2}}\left[\exp \left(-\frac{i\left(\mathbf{r}^{\prime}-\mathbf{v} t^{\prime}\right)^{2}}{2\left(t^{\prime}-t\right)}\right) c_{1}\left(t^{\prime}\right)+\exp \left(-\frac{i\left(\mathbf{r}^{\prime}+\mathbf{v} t^{\prime}\right)}{2\left(t^{\prime}-t\right)}\right) c_{2}\left(t^{\prime}\right)\right]
$$


причем

$$
\begin{aligned}
\left.\psi\right|_{\mathbf{r} \rightarrow \mathbf{v} t} & =c_{1}(t)\left(\frac{1}{|\mathbf{r}-\mathbf{v} t|}-\kappa_{1}+\frac{i \mathbf{v}(\mathbf{r}-\mathbf{v} t)}{|\mathbf{r}-\mathbf{v} t|}\right) \\
\left.\psi\right|_{\mathbf{r} \rightarrow-\mathbf{v} t} & =c_{2}(t)\left(\frac{1}{|\mathbf{r}+\mathbf{v} t|}-\kappa_{2}-\frac{i \mathbf{v}(\mathbf{r}+\mathbf{v} t)}{|\mathbf{r}+\mathbf{v} t|}\right)
\end{aligned}
$$

Пусть $g_{1,2} e^{i v^{2} t / 2}=c_{1,2}(t)$. Тогда из $(23)$ следуют уравнения

$$
\begin{aligned}
& g_{1}(t)=-\frac{2 i}{\kappa_{1} \sqrt{2 \pi i}} \int_{t}^{\infty} \frac{\dot{g}_{1}\left(t^{\prime}\right) d t^{\prime}}{\sqrt{t^{\prime}-t}}-\frac{i}{\kappa_{2} \sqrt{2 \pi i}} \int_{t}^{\infty} \frac{g_{2}\left(t^{\prime}\right) \exp \left(-\frac{2 i v^{2} t t^{\prime}}{t^{\prime}-t}\right) d t^{\prime}}{\left(t^{\prime}-t\right)^{3 / 2}}, \\
& g_{2}(t)=-\frac{i}{\kappa_{1} \sqrt{2 \pi i}} \int_{t}^{\infty} \frac{g_{1}\left(t^{\prime}\right) \exp \left(-\frac{2 i v^{2} t t^{\prime}}{t^{\prime}-t}\right) d t^{\prime}}{\left(t^{\prime}-t\right)^{3 / 2}}-\frac{2 i}{\kappa_{2} \sqrt{2 \pi i}} \int_{t}^{\infty} \frac{\dot{g}_{2}\left(t^{\prime}\right) d t^{\prime}}{\sqrt{t^{\prime}-t}}
\end{aligned}
$$

Положим $g_{1,2}\left(\tau^{-1}\right)=h_{1,2}(\tau) \sqrt{\tau}$, тогда для образов Лапласа $H_{1,2}(p)$ функций $h_{1,2}(\tau)$ можно получить уравнения

$$
\begin{aligned}
& i \kappa_{1} q H_{1}(q)=H_{1}(q)+q \frac{d H}{d q}-\frac{\kappa_{1}}{\kappa_{2}} \frac{1}{2 v} e^{-2 v q} \frac{d H_{2}}{d q}+\frac{\kappa_{1}}{\kappa_{2}} e^{-2 v q} H_{2}, \\
& i \kappa_{2} q H_{2}(q)=H_{2}(q)+q \frac{d H_{2}}{d q}-\frac{\kappa_{2}}{\kappa_{1}} \frac{1}{2 v} e^{-2 v q} \frac{d H_{1}}{d q}+\frac{\kappa_{2}}{\kappa_{1}} e^{-2 v q} H_{1} .
\end{aligned}
$$

Система (24) не имеет, по-видимому, достаточно простого решения, выражаемого через какие-либо известные функции.

При помощи соотношения (23) решение для $\psi$-функции может быть представлено в виде достаточно сложных интегралов от функций $H_{1,2}(q)$.

\section{Список литературы}

[1] G. Breit. Ann. Phys. 1965. V. 34. P. 377; G. Herling, Y. Nishida. Ann. Phys. 1965. V. 34. P. 400; Y. Nishida. Ann. Phys. 1965. V. 34. P. 415.

[2] W. Däppen. J. Phys. B. 1977. V. 10. P. 2399.

[3] С. К. ЖДанов, А. С. Чихачев. Нейтрализация и перезарядка частиц в одномерной модели. Деп. ВИнИТИ № 2221-74. М.: ВИНИТИ; H. Danared. J. Phys. В. 1984. V. 17. Р. 2619; E. А. Соловьев. ТМФ. 1976. Т. 28. С. 240.

[4] J. H. Macek, S. Yu. Ovchinnikov, E. A. Solov'ev. Phys. Rev. A. 1999. V. 60. № 2. P. 1140; M. J. Rakovic, E. A. Solov'ev. Phys. Rev. A. 1990. V. 41. № 1. P. 3635; J. Burgdörfer, J. Wang, A. Barany. Phys. Rev. A. 1988. V. 38. P. 4919; J. Wang, J. Burgdörfer, A. Barany. Phys. Rev. A. 1991. V. 43. P. 4036.

[5] С. К. Жданов, А. С. Чихачев. ДАН СССР. 1974. Т. 218. С. 1323.

[6] В. И. Манько, А. С. Чихачев. ЖЭТФ. 1998. Т. 113. С. 606.

[7] G. Scheitler, M. Kleber. Phys. Rev. A. 1990. V. 42. P. 55; В. И. Манько, А. С. Чихачев. ЯФ. 2001. Т. 64. № 8. С. 1533; А. С. Чихачев. ЖЭТФ. 2004. Т. 125. С. 1012.

[8] Ю. Н. Демков, В. Н. Островский. Метод потенциалов нулевого радиуса действия в атомной физике. Л.: Изд-во ЛГУ, 1975.

Поступила в редакцию 9.II.2005 г., после доработки 15.IV.2005 г. 